\title{
Sitagliptin: a potential drug for the treatment of COVID-19?
}

\author{
SANAA K. BARDAWEEL ${ }^{1, *}$ \\ RIMA HAJJO2 \\ DIMA A. SABBAH ${ }^{2}$ \\ ${ }^{1}$ Department of Pharmaceutical Sciences \\ School of Pharmacy, University \\ of Jordan, Amman 11942, Jordan \\ ${ }^{2}$ Department of Pharmacy, Faculty \\ of Pharmacy, Al-Zaytoonah University \\ of Jordan, P.O. Box 130, Amman 11733 \\ Jordan
}

\begin{abstract}
Recently, an outbreak of a fatal coronavirus, SARS-CoV-2, has emerged from China and is rapidly spreading worldwide. Possible interaction of SARS-CoV-2 with DPP4 peptidase may partly contribute to the viral pathogenesis. An integrative bioinformatics approach starting with mining the biomedical literature for high confidence DPP4-protein/gene associations followed by functional analysis using network analysis and pathway enrichment was adopted. The results indicate that the identified DPP4 networks are highly enriched in viral processes required for viral entry and infection, and as a result, we propose DPP4 as an important putative target for the treatment of COVID-19. Additionally, our protein-chemical interaction networks identified important interactions between DPP4 and sitagliptin. We conclude that sitagliptin may be beneficial for the treatment of COVID-19 disease, either as monotherapy or in combination with other therapies, especially for diabetic patients and patients with pre-existing cardiovascular conditions who are already at higher risk of COVID-19 mortality.
\end{abstract}

Keywords: SARS-CoV-2, COVID-19, DPP4, ACE2, sitagliptin, drug repurposing, CXCL10

In December 2019, an outbreak of fatal coronavirus, COVID-19, has emerged from China and is rapidly spreading worldwide due to its high transmission rate $(1,2)$. This new coronavirus is named SARS-CoV-2 which appears to have originated from wild animals and birds (2). To date, more than a million cases have been reported globally making COVID-19 a major health issue. Currently, huge efforts are being made towards the development of vaccines and antiviral drugs for the treatment of COVID-19.

As the coronavirus pandemic rages, drug discovery and development become even more challenging. Research focus has been directed to repurposing already existing, FDA-approved drugs to treat critically ill patients. Drug repurposing is an efficient approach to provide therapeutic moieties with known safety profiles and predicted side-effects for the treatment of evolving diseases. In this context, the antimalarial drug chloroquine and its hydroxylated form had demonstrated apparent effectiveness in the treatment of COVID-19-associated pneumonia in clinical trials $(3,4)$. In addition, several other drugs have been

\footnotetext{
*Correspondence; e-mail: S.Bardaweel@ju.edu.jo
} 
reported for their potential efficacy against COVID-19, such as remdesivir, lopinavir, ribavirin and ritonavir $(4,5)$.

Lately, the complete genome sequence of SARS-CoV-2 was published and deposited in the NCBI database with reference number MN908947.3. The availability of the full genome sequence provided a clue for understanding the possible patterns of viral protein glycosylation and proposed mechanisms for viral-host interactions (6). According to the latest published reports, and analogous to most other coronaviruses, the outer membrane spike glycoprotein appears to be the key viral protein that interacts with host cellular targets $(6,7)$.

Interestingly, a model for the homo-trimer structure of COVID-19 spike glycoprotein has been published recently (6). According to the study findings, the S1 domain of COVID-19 spike glycoprotein demonstrates evident interaction with the human CD26, also named dipeptidyl peptidase-4 (DPP4), a crucial immunomodulatory protein for hijacking and virulence (6).

Remarkably, the SARS-CoV-2 spike protein shares a $31.9 \%$ sequence identity with the spike protein present in the Middle East Respiratory Syndrome Corona Virus (MERS$\mathrm{CoV}$ ), another human coronavirus that was first identified in humans in 2012 (8). MERS$\mathrm{CoV}$ infects cells through the interaction of its spike protein with the DPP4 receptor found on macrophages (8). The DPP4 receptor was then identified as one of the most important target proteins for antiviral drug development directed against MERS-CoV (8). Indeed, it has been demonstrated that the MERS-CoV spike protein activity suppressed macrophage responses via DPP4-mediated stimulation of IRAK-M and PPAR $\gamma$ (9), and the observed suppression was reversed by the DPP4 inhibitor sitagliptin.

Sitagliptin, a dipeptidyl peptidase-4 inhibitor, is known for its antidiabetic, immunoregulatory, anti-inflammatory, and beneficial cardiometabolic effects $(10,11)$. Interestingly, sitagliptin diminished hepatitis $C$ virus replication in a diabetic patient by an unknown mechanism (12). In addition, sitagliptin was shown to suppress the production of interferon gamma-induced protein 10 (CXCL10) chemokine in AIDS patients (13). Very recently, CXCL10 chemokine has been detected at a high level of expression in the lung bronchoalveolar microenvironment of COVID-19 patients (14).

In the view of the current public health catastrophe and considering the potential molecular interactions between COVID-19 spike protein and DPP4, we suggest that DPP4 inhibition may be potentially useful as an alternative for the treatment of COVID-19 disease especially in diabetic patients and patients with pre-existing cardiovascular conditions who are already at higher risk of COVID-19 mortality.

\section{METHODS}

\section{Database mining to identify DPP4 functional partners}

A systematic search for human DPP4's nearest neighbor (NN) proteins was conducted in Cytoscape (15) version 3.7.2 using the STRING (16) protein query application. All retrieved protein-protein interactions, including both physical and functional interactions were retrieved from popular databases such as MINT (17), HPRD (18), BIND (19), DIP (20), BioGRID (21), KEGG (22), Reactome (23), EcoCyc (24), NCI-Nature Pathway Interaction Database (25), and Gene Ontology (GO) (26) protein complexes. 


\section{Network building}

Network building tools in Cytoscapes version 3.7.2 were used to generate DPP4-protein interaction networks.

\section{Pathway enrichment analysis}

Functional enrichment tools in Cytoscape were applied to conduct an over-representation analysis of DPP4 and its NN proteins in biological pathways to determine whether DPP4's NN protein network is important in viral and immune processes.

\section{Protein structure analysis}

The crystal structure of DPP-IV (PDB ID: 1X70) (27) co-crystallized with sitagliptin (715) was retrieved from the RCSB Protein Data Bank (28).

\section{RESULTS AND DISCUSSION}

Viruses require receptors on the surface of the target cell to launch infection. There has been a huge focus in the reported literature on the ability of both SARS-CoV-2 and SARS-CoV to bind to angiotensin-converting enzyme II (ACE2) protein in order to invade the host cells $(29,30)$. Since the outbreak, many studies were published reporting the dis-

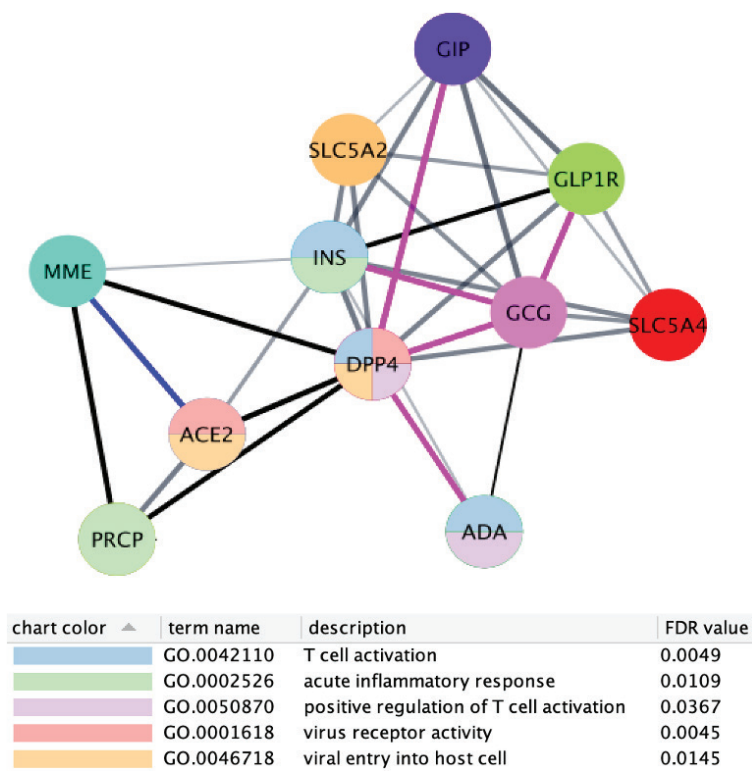

Fig. 1. DPP4 nearest neighbor proteins. The nodes are color-coded using a split pie chart coloring scheme indicating enriched GO terms involved in viral, inflammatory and immune processes. All details about the complete list of enriched pathways, network genes, gene sets, background genes and enrichments are found in Supplementary Table S1. 
tribution of ACE2 receptor in the different types of human cells, such as lung, liver, kidney, and colon (31) and suggesting that SARS-CoV-2 may infect different organs in the human body. Nevertheless, the main target cell for SARS-CoV-2 entry, lung alveolar type 2 (AT2) cells, were shown to express rather low levels of ACE2 (31) suggesting a possible existence of co-membrane proteins facilitating host entry and infection.

Our results showed that DPP4 interacts with several proteins that are important for viral processes and immune responses including ACE2, which implies a cross-talk between the two proteins that warrants additional investigation. We identified supporting evidence from the biomedical literature in two recent reports which highlighted the possible role of DPP4 as an alternative pathway for SARS-CoV-2 entry and infection $(6,32)$. Indeed, DPP4 has been suggested as a candidate co-receptor for SARS-CoV-2 entry, displaying analogous expression patterns with ACE2 across 13 human tissues (32).

A network of DPP4's functional NN proteins was generated using the STRING protein interaction app in Cytoscape as illustrated in Fig. 1. In this network, nodes are identified as NN proteins and edges are indicating the type of evidence for the mined protein-protein interactions. The network generating algorithm included interactions from text and database mining, direct experiments, co-expression, and co-occurrence.

Additionally, DPP4 and its NN proteins were used as a query for an automated overrepresentation analysis conducted across all annotated biological pathways included in STRING. This analysis highlighted enriched GO processes and GO functions associated with viral, inflammatory and immune processes. The most important enriched terms with false discovery rates (FDRs) lower than 0.05 were: "virus receptor activity", "viral entry into host cell", "T-cell receptor", "positive regulation of T cell activation", and "acute inflammatory response".

Interestingly, supporting the idea that DPP4 inhibition may be beneficial as a treatment option for COVID-19, recent studies have demonstrated that DPP4 inhibitors interact

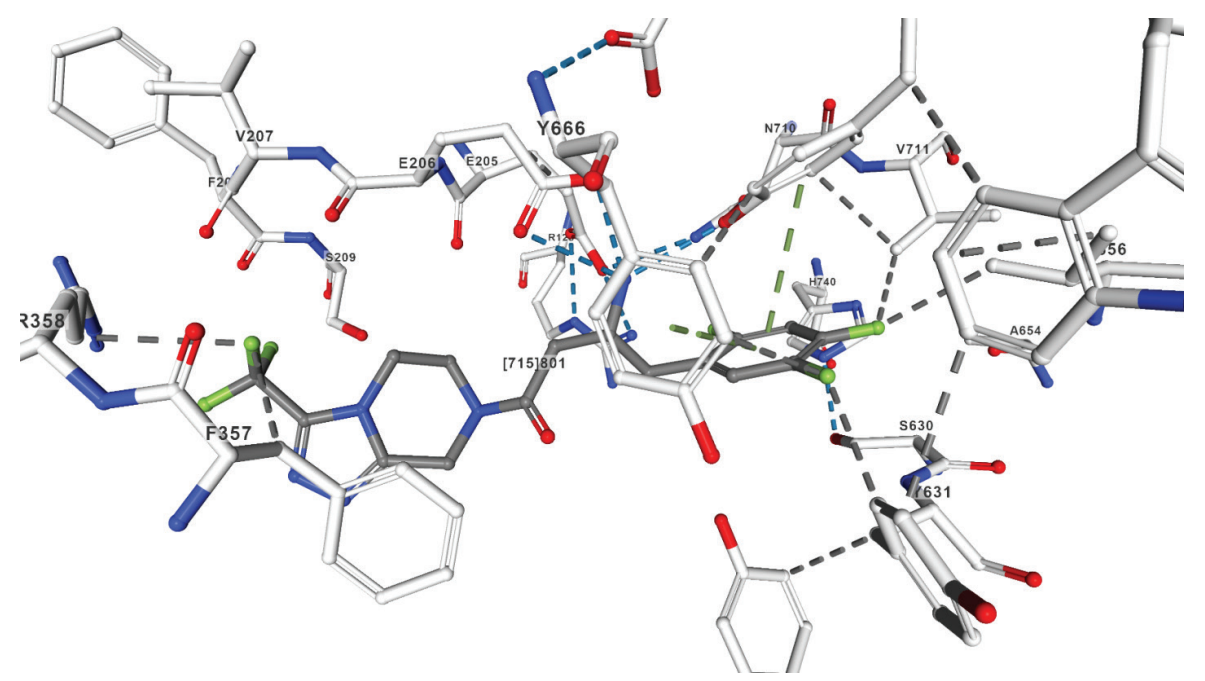

Fig. 2. Crystal structure of dipeptidyl peptidase IV (DPP4, DPP-IV) (PDB ID: 1X70) (27) in complex with sitagliptin (715). The picture is created by NGL $(28,34)$. 
with ACE2 at a relatively reasonable binding energy (33). Moreover, in vivo studies showed that sitagliptin could inhibit ACE activity and reduce angiotensin II levels in rats (33).

In order to identify the structural basis of the binding of sitagliptin in DPP4, we adopted the crystal structure of DPP4 (PDB ID: 1X70) co-crystallized with sitagliptin (715) Fig. 2 (27).

The hydrophilic and hydrophobic residues occupy DPP4 binding cleft. The binding pocket of DPP4 encloses E205, E206, V207, F208, S209, F357, R358, Y547, S630, Y631, V656, W659, Y662, D663, Y666, and H740. Interestingly, the hydrophilic and hydrophobic exposed surfaces of sitagliptin agree with the nearby residues. The aromatic residues furnish aromatic ( $\pi-\pi$ stacking) whereas hydrophobic amino acids mediate hydrophobic interaction. On the contrary, the hydrophilic residues provide dipole-dipole, hydrogen-bonding, and ion-dipole interactions. And the acidic and basic residues offer an electrostatic (ionic) bond.

Furthermore, to investigate the functional effects resulting from DPP4 inhibition by sitagliptin, we built a new network for DPP4 using all reported protein targets for sitagliptin as seeds, which included DPP4, CYP2C8, FASLG, HMGCR and SLC22A8. The resulting network, which consisted of all of the above-mentioned sitagliptin's protein targets seed nodes, was then expanded with the addition of 20 first-shell protein interactions, and 20 -second shell protein interactions. All proteins in the extended network were then used for the enrichment analysis in STRING. Results for pathways involving viral processes are shown in Table I and all details about the complete list of enriched pathways, network genes, gene sets, background genes and enrichments are found in supplementary Table S2.

Our extended network highlighted an important role of caveolin-1 (CAV1) in sitagliptin's functional interactions (Fig. 3). In fact, caveolin-1 may act as a scaffolding protein within caveolar membranes; it interacts directly with G-protein alpha subunits and can functionally regulate their activity. It is also involved in the co-stimulatory signal essential for the T-cell receptor (TCR)-mediated T-cell activation. Binding of CAV1 to DPP4 induces T-cell proliferation and NF-kappa-B activation in a T-cell receptor CD3-dependent manner (35). Noteworthy, the endocytic pathway and the autophagy process are key elements in viral infection and have gained considerable attention over the last years. Hence, the

Table I. Enriched viral processes and pathways in the sitagliptin drug target network

\begin{tabular}{ccccc}
\hline Enriched pathway & Category & $\begin{array}{c}\text { Number of } \\
\text { genes }\end{array}$ & $\begin{array}{c}\text { Background } \\
\text { genes }\end{array}$ & FDR \\
\hline Viral mRNA translation & Reactome pathway & 18 & 86 & $2.88 \mathrm{E}-26$ \\
Viral process & GO process & 11 & 571 & $5.15 \mathrm{E}-06$ \\
Viral life cycle & GO process & 7 & 166 & $6.40 \mathrm{E}-06$ \\
ISG15 antiviral mechanism & Reactome pathway & 3 & 69 & 0.0016 \\
Viral entry into the host cell & GO process & 3 & 92 & 0.0091 \\
Viral myocarditis & KEGG pathway & 2 & 56 & 0.0369 \\
Host-virus interaction & Keywords & 5 & 432 & 0.0386 \\
\hline
\end{tabular}

FDR - false discovery rate 
endocytic pathway, embracing endosome and lysosome, was often suggested as a hot target for the development of new therapeutic approaches in tackling coronaviruses' infections (35). Nevertheless, and despite the general agreement on the endocytic pathway role in viral entry, there are several contradicting reports with major discrepancies in the precise mechanism via which this pathway mediates SARS-CoV infections (35).

For example, Wang et al. (36) reported that SARS-CoVs use clathrin- and caveolae-independent endocytic pathway as the main mechanism for viral entry, whereas other studies demonstrate that SARS-CoV cell entry is chiefly facilitated by the clathrin-dependent pathway (37).

Currently, the precise mechanism of cell entry and the involvement of the endocytic pathway of the new evolving SARS-CoV-2 have not been described thoroughly. However,

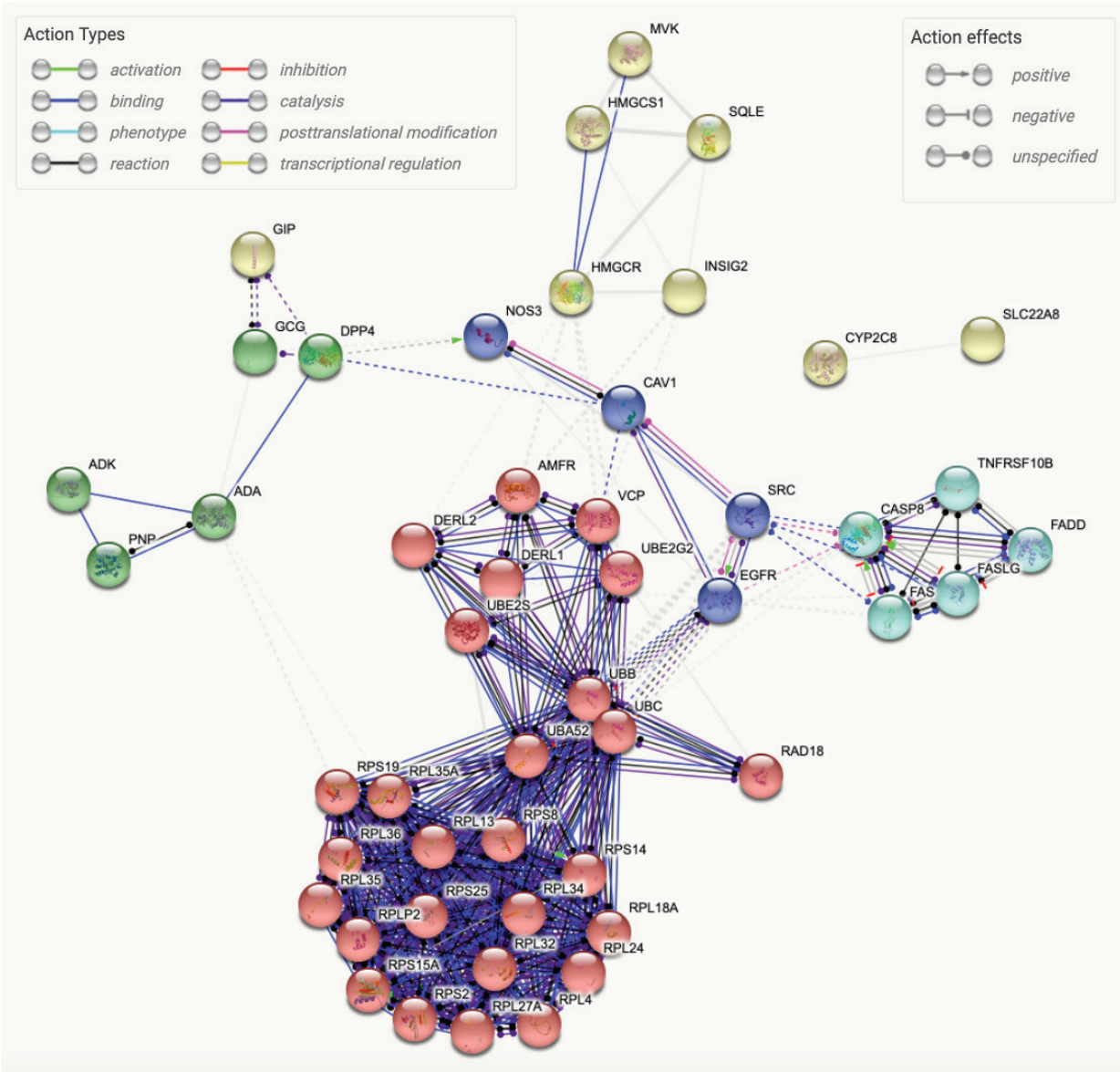

Fig. 3. Expanded protein interactions network of sitagliptin's drug targets. The nodes were clustered using K-means clustering setting the number of clusters to 5 and colored as red, green, violet, cyan and yellow. Edges are colored according to their action types explained in the figure. 
there is growing evidence supporting that SARS-CoV-2 utilizes the same entry receptor that SARS-CoV uses, the ACE2 membrane protein $(29,30)$. In addition, it has been reported that SARS-CoV-2 is sensitive to the inhibitory effects of the antimalarial drug chloroquine, a compound with lysosomotropic properties. Therefore, it is likely that the emerging SARS-CoV-2 utilizes more than one pathway for cell entry such as the endocytic pathway used by other SARS-CoVs and the ACE2 receptor entry mechanism, both of which may cross-talk with the multifunctional DPP4 protein.

Interestingly, a similar case scenario has been reported with the human immunodeficiency virus (HIV) where the CD4 antigen is shown to be essential for the binding of the virus but is not enough for efficient viral entry and infection (38). Later, the CD26/DPP4 has been demonstrated as a crucial cofactor for efficient viral entry and the co-expression of human CD4 and CD26/DPP4 in murine NIH 3T3 cells resulted in evident infection by HIV (38). It could be that emerging SARS-CoV-2 utilizes similar patterns of ACE2/DPP4 crosstalk and that specific inhibitors that block the function of DPP4 may be effective therapeutic agents in COVID-19. Further investigation into the inhibitory effects of sitagliptin on viral entry and infection should shed light on possible therapeutic avenues for the treatment of COVID-19.

\section{CONCLUSIONS}

Taken together, we conclude that the use of DPP4 inhibitors such as sitagliptin may be a potential treatment of COVID-19 disease, either as monotherapy or in the combination with other therapies, especially for diabetic patients and patients with pre-existing cardiovascular conditions who are already at higher risk of COVID-19 mortality.

Acknowledgements. - This work has been funded by The Scientific Research Deanship at The University of Jordan. The funding body had no role in the design, analysis or interpretation of this study.

Supplementary data available upon request.

Acronyms, symbols, abbreviations. - ACE2 - angiotensin converting enzyme II, AT2 - lung alveolar type 2, BIND - Biomolecular Interaction Network Database, BioGRID - Biological General Repository for Interaction Datasets, CAV1 - caveolin-1, COVID-19 - Coronavirus disease 2019, CXCL10 interferon gamma-induced protein 10, CYP2C 8 - cytochrome P450 family 2 subfamily C member 8 , DIP - Database of Interacting Proteins, DPP4 - dipeptidyl peptidase-4, EcoCyc - Encyclopedia of E. coli Genes and Metabolism, FASLG - Fas cell surface death receptor ligand, FDRs - false discovery rates, GO - gene ontology, HIV - human immunodeficiency virus, HMGCR - hydroxymethylglutaryl-CoA Reductase, IRAK-M - IL-1 receptor-associated kinase M, KEGG - Kyoto Encyclopedia of Genes and Genomes, MERS - Middle East Respiratory Syndrome Corona Virus, NCBI - National Center for Biotechnology Information, NN - nearest network, PDB - protein data bank, PPAR $\gamma$ - peroxisome proliferator-activated receptor gamma, RCSB - Research Collaboratory for Structural Bioinformatics, SARS-Cov2 - severe acute respiratory syndrome coronavirus 2, SLC22A8 - solute carrier family 22 member 8, STRING - Search Tool for the Retrieval of Interacting Genes/Proteins, TCR - T-cell receptor.

\section{REFERENCES}

1. C. Huang, Y. Wang, X. Li, L. Ren, J. Zhao, Y. Hu, L. Zhang, G. Fan, J. Xu and X. Gu, Clinical features of patients infected with 2019 novel coronavirus in Wuhan, China, Lancet 395 (2020) 497-506; https://doi.org/10.1016/S0140-6736 
2. H. Lu, C. W. Stratton and Y. W. Tang, Outbreak of pneumonia of unknown etiology in Wuhan China: the mystery and the miracle, J. Med. Virol. 92 (2020) 401-402; https://doi.org/10.1002/jmv.25678

3. P. Colson, J. M. Rolain and D. Raoult, Chloroquine for the 2019 novel coronavirus SARS Cov2, Int. J. Antimicrob. Agents 55 (2020) Article ID 105923 (3 pages); https://doi.org/10.1016/j.ijantimicag.2020.105923

4. M. Wang, R. Cao, L. Zhang, X. Yang, J. Liu, M. Xu, Z. Shi, Z. Hu, W. Zhong and G. Xiao, Remdesivir and chloroquine effectively inhibit the recently emerged novel coronavirus (2019-nCoV) in vitro, Cell Res. 30 (2020) 269-271; https://doi.org/10.1038/s41422-020-0282-0

5. W. Ko, J. Rolain, N. Lee, P. Chen, C. Huang and P. Lee, Arguments in favour of remdesivir for treating SARS-CoV-2 infections, Int. J. Antimicrob. Agents (2020) Article ID 105933 (4 pages); https:// doi.org/10.1016/j.ijantimicag.2020.105933

6. N. Vankadari and J. A. Wilce, Emerging WuHan (COVID-19) coronavirus: glycan shield and structure prediction of spike glycoprotein and its interaction with human CD26, Emerg. Microbes Infect. 9 (2020) 601-604; https://doi.org/10.1080/22221751.2020.1739565

7. W. Song, M. Gui, X. Wang and Y. Xiang, Cryo-EM structure of the SARS coronavirus spike glycoprotein in complex with its host cell receptor ACE2, PLoS Pathog. 14 (2018) e1007236 (19 pages); https://doi.org/10.1371/journal.ppat.1007236

8. Y. Zhou, Y. Hou, J. Shen, Y. Huang, W. Martin and F. Cheng, Network-based drug repurposing for novel coronavirus 2019-nCoV/SARS-CoV-2, Cell Discov. 6 (2020) 1-18; https://doi.org/10.1038/s41421020-0153-3

9. A. A. Al-Qahtani, K. Lyroni, M. Aznaourova, M. Tseliou, M. R. Al-Anazi, M. N. Al-Ahdal, S. Alkahtani, G. Sourvinos and C. Tsatsanis, Middle east respiratory syndrome corona virus spike glycoprotein suppresses macrophage responses via DPP4-mediated induction of IRAK-M and PPAR $\gamma$, Oncotarget 8 (2017) 9053-9066; https://doi.org/10.18632/oncotarget.14754

10. A. Makdissi, H. Ghanim, M. Vora, K. Green, S. Abuaysheh, A. Chaudhuri, S. Dhindsa and P. Dandona, Sitagliptin exerts an antinflammatory action, J. Clin. Endocrinol. Metab. 97 (2012) 3333 3341; https://doi.org/10.1210/jc.2012-1544

11. J. R. Ussher and D. J. Drucker, Cardiovascular biology of the incretin system, Endocr. Rev. 33 (2012) 187-215; https://doi.org/10.1210/er.2011-1052

12. H. Yanai, Dipeptidyl peptidase-4 inhibitor sitagliptin significantly reduced hepatitis $C$ virus replication in a diabetic patient with chronic hepatitis $C$ virus infection, Hepatobiliary Pancreat. Dis. Int. 13 (2014) 556; https://doi.org/10.1016/S1499-3872(14)60308-8

13. M. P. Dubé, E. S. Chan, J. E. Lake, B. Williams, J. Kinslow, A. Landay, R. W. Coombs, M. FlorisMoore, H. J. Ribaudo and K. E. Yarasheski, A randomized, double-blinded, placebo-controlled trial of sitagliptin for reducing inflammation and immune activation in treated and suppressed human immunodeficiency virus infection, Clin. Infect. Dis. 69 (2019) 1165-1172; https://doi. org/10.1093/cid/ciy1051

14. M. Liao, Y. Liu, J. Yuan, Y. Wen, G. Xu, J. Zhao, L. Chen, J. Li, X. Wang, F. Wang, L. Liu, S. Zhang and Z. Zhang, The landscape of lung bronchoalveolar immune cells in COVID-19 revealed by single-cell RNA sequencing, medRxiv preprint, posted February 26, 2020 (23 pages); https://doi. org/10.1101/2020.02.23.20026690

15. P. Shannon, A. Markiel, O. Ozier, N. S. Baliga, J. T. Wang, D. Ramage, N. Amin, B. Schwikowski and T. Ideker, Cytoscape: a software environment for integrated models of biomolecular interaction networks, Genome Res. 13 (2013) 2498-2504; https://doi.org/10.1101/gr.1239303

16. D. Szklarczyk, A. L. Gable, D. Lyon, A. Junge, S. Wyder, J. Huerta-Cepas, M. Simonovic, N. T. Doncheva, J. H. Morris, P. Bork, L. J. Jensen and C. V. Mering, STRING v11: protein-protein association networks with increased coverage, supporting functional discovery in genome-wide experimental datasets, Nucleic Acids Res. 47 (2019) D607-D613; https://doi.org/10.1093/nar/gky1131 
17. A. Chatr-Aryamontri, A. Ceol, L. M. Palazzi, G. Nardelli, M. V. Schneider, L. Castagnoli and G. Cesareni, MINT: the Molecular INTeraction database, Nucleic Acids Res. 35 (2007) D572-D574; https://doi.org/10.1093/nar/gk1950

18. S. Peri, J. D. Navarro, T. Z. Kristiansen, R. Amanchy, V. Surendranath, B. Muthusamy, T. Gandhi, K. Chandrika, N. Deshpande and S. Suresh, Human protein reference database as a discovery resource for proteomics, Nucleic Acids Res. 32 (2004) D497-D501; https://doi.org/10.1093/nar/gkh070

19. R. Wang, X. Fang, Y. Lu and S. Wang, The PDBbind database: Collection of binding affinities for protein- ligand complexes with known three-dimensional structures, J. Med. Chem. 47 (2004) 2977-2980; https://doi.org/10.1021/jm0305801

20. L. Salwinski, C. S. Miller, A. J. Smith, F. K. Pettit, J. U. Bowie and D. Eisenberg, The database of interacting proteins: 2004 update, Nucleic Acids Res. 32 (2004) D449-D451; https://doi.org/10.1093/ nar/gkh086

21. B. J. Breitkreutz, C. Stark, T. Reguly, L. Boucher, A. Breitkreutz, M. Livstone, R. Oughtred, D. H. Lackner, J. Bähler and V. Wood, The BioGRID interaction database: 2008 update, Nucleic Acids Res. 36 (2008) D637-D640; https://doi.org/10.1093/nar/gkm1001

22. M. Kanehisa, M. Araki, S. Goto, M. Hattori, M. Hirakawa, M. Itoh, T. Katayama, S. Kawashima, S. Okuda and T. Tokimatsu, KEGG for linking genomes to life and the environment, Nucleic Acids Res. 36 (2007) D480-D484; https://doi.org/10.1093/nar/gkm882

23. D. Croft, A. F. Mundo, R. Haw, M. Milacic, J. Weiser, G. Wu, M. Caudy, P. Garapati, M. Gillespie and M. R. Kamdar, The Reactome pathway knowledgebase, Nucleic Acids Res. 42 (2014) D472D477; https://doi.org/10.1093/nar/gkt1102

24. I. M. Keseler, J. Collado-Vides, S. Gama-Castro, J. Ingraham, S. Paley, I. T. Paulsen, M. Peralta-Gil and P. D. Karp, EcoCyc: a comprehensive database resource for Escherichia coli, Nucleic Acids Res. 33 (2005) D334-D337; https://doi.org/10.1093/nar/gkq1143

25. S. Krupa, K. Anthony, J. Buchoff, M. Day, T. Hannay and C. Schaefer, Pathway Interaction Database: A cell signaling resource, Nature 446 (2007) 153-158; https://doi.org/10.1038/npre.2007.1311.1

26. Gene Ontology Consortium, The Gene Ontology (GO) database and informatics resource, Nucleic Acids Res. 32 (2004) D258-D261; https://doi.org/10.1093/nar/gkh036

27. D. Kim, L. Wang, M. Beconi, G. J. Eiermann, M. H. Fisher, H. He, G. J. Hickey, J. E. Kowalchick, B. Leiting and K. Lyons, (2R)-4-oxo-4-[3-(trifluoromethyl)-5,6-dihydro[1,2,4]triazolo[4,3-a] pyrazin7(8H)-yl]-1-(2,4,5-trifluorophenyl)butan-2-amine: a potent, orally active dipeptidyl peptidase IV inhibitor for the treatment of type 2 diabetes, J. Med. Chem. 48 (2005) 141-151; https://doi.org/10.1021/ jm0493156

28. H. Berman, K. Henrick and H. Nakamura, Announcing the worldwide protein data bank, Nat. Struct. Mol. Biol. 10 (2003) 980; https://doi.org/10.1038/nsb1203-980

29. R. Lu, X. Zhao, J. Li, P. Niu, B. Yang, H. Wu, W. Wang, H. Song, B. Huang and N. Zhu, Genomic characterisation and epidemiology of 2019 novel coronavirus: implications for virus origins and receptor binding, Lancet 395 (2020) 565-574; https://doi.org/10.1016/S0140-6736(20)30251-8

30. M. Hoffmann, H. Kleine-Weber, N. Krüger, M. Mueller, C. Drosten and S. Pöhlmann, The novel coronavirus 2019 (2019-nCoV) uses the SARS-coronavirus receptor ACE2 and the cellular protease TMPRSS2 for entry into target cells, bioRxiv preprint, posted January 31, 2020 (23 pages); https:// doi.org/10.1101/2020.01.31.929042

31. X. Zou, K. Chen, J. Zou, P. Han, J. Hao and Z. Han, Single-cell RNA-seq data analysis on the receptor ACE2 expression reveals the potential risk of different human organs vulnerable to 2019-nCoV infection, Front. Med. (2020) (8 pages); https://doi.org/10.1007/s11684-020-0754-0

32. F. Qi, S. Qian, S. Zhang and Z. Zhang, Single cell RNA sequencing of 13 human tissues identify cell types and receptors of human coronaviruses, Biochem. Biophys. Res. Commun. (2020) (7 pages); https://doi.org/10.1016/j.bbrc.2020.03.044 
33. M. Abouelkheir and T. H. El-Metwally, Dipeptidyl peptidase-4 inhibitors can inhibit angiotensin converting enzyme, Eur. J. Pharmacol. 862 (2019) Article ID 172638; https://doi.org/10.1016/j. ejphar.2019.172638

34. A. S. Rose, A. R. Bradley, Y. Valasatava, L. M. Duarte, A. Prlić and P. W. Rose, NGL viewer: webbased molecular graphics for large complexes, Bioinformatics 34 (2018) 3755-3758; https://doi. org/10.1093/bioinformatics/bty419

35. N. Yang and H.-M. Shen, Targeting the endocytic pathway and autophagy process as a novel therapeutic strategy in COVID-19, Int. J. Biol. Sci. 16 (2020) 1724-1731; https://doi.org/10.7150/ ijbs. 45498

36. H. Wang, P. Yang, K. Liu, F. Guo, Y. Zhang, G. Zhang and C. Jiang, SARS coronavirus entry into host cells through a novel clathrin-and caveolae-independent endocytic pathway, Cell Res. 18 (2008) 290-301; https://doi.org/10.1038/cr.2008.15

37. Y. Inoue, N. Tanaka, Y. Tanaka, S. Inoue, K. Morita, M. Zhuang, T. Hattori and K. Sugamura, Clathrin-dependent entry of severe acute respiratory syndrome coronavirus into target cells expressing ACE2 with the cytoplasmic tail deleted, J. Virol. 81 (2007) 8722-8729; https://doi.org/ 10.1128/JVI.00253-07

38. C. Callebaut, B. Krust, E. Jacotot and A. G. Hovanessian, T cell activation antigen, CD26, as a cofactor for entry of HIV in CD4+ cells, Science 262 (1993) 2045-2050; https://doi.org/10.1126/science.7903479 